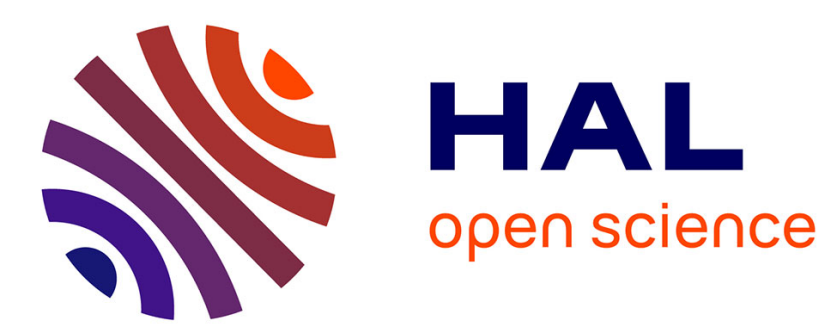

\title{
Lithium intercalated graphite: experimental Compton profile for stage one
}

\author{
G. Loupias, J. Chomilier, D. Guérard
}

\section{To cite this version:}

G. Loupias, J. Chomilier, D. Guérard. Lithium intercalated graphite: experimental Compton profile for stage one. Journal de Physique Lettres, 1984, 45 (7), pp.301-306. 10.1051/jphyslet:01984004507030100 . jpa-00232347

\section{HAL Id: jpa-00232347 https://hal.science/jpa-00232347}

Submitted on 1 Jan 1984

HAL is a multi-disciplinary open access archive for the deposit and dissemination of scientific research documents, whether they are published or not. The documents may come from teaching and research institutions in France or abroad, or from public or private research centers.
L'archive ouverte pluridisciplinaire HAL, est destinée au dépôt et à la diffusion de documents scientifiques de niveau recherche, publiés ou non, émanant des établissements d'enseignement et de recherche français ou étrangers, des laboratoires publics ou privés. 
Classification

Physics Abstracts

78.70

\title{
Lithium intercalated graphite : experimental Compton profile for stage one
}

\author{
G. Loupias, J. Chomilier \\ Laboratoire de Cristallographie, Université Paris 6, Tour 16, 4, place Jussieu, 75230 Paris Cedex 05, \\ France \\ and LURE, Université Paris-Sud, Bât. 209C, 91405 Orsay Cedex, France
}

and D. Guérard

Laboratoire de Chimie Minérale Appliquée, Université Nancy 1, 54506 Vandouvre Les Nancy Cedex, France

(Reçu le 25 novembre 1983, accepté le 14 février 1984)

\begin{abstract}
Résumé. - La distribution d'impulsions électroniques du composé intercalaire du graphite de premier stade $\mathrm{LiC}_{6}$ est mesurée par diffusion inélastique de photons $\mathrm{X}$ et comparée à celle du graphite, grâce au spectromètre à haute résolution de LURE-DCI. La différence des profils Compton est compatible avec un transfert total de l'électron de conduction du lithium vers une bande $\pi$ comme le prévoit un modèle de bandes rigides. Mais ce modèle est insuffisant pour expliquer tous les aspects du profil différence qui sont en fait l'illustration de la modification électronique due à l'insertion.
\end{abstract}

\begin{abstract}
Electron momentum distribution of the first stage lithium intercalated graphite $\left(\mathrm{LiC}_{6}\right)$ is measured by $\mathrm{X}$-ray inelastic scattering and compared to the graphite one, using the high resolution spectrometer from LURE-DCI synchrotron source. The Compton profile difference is consistent with a total transfer of the lithium conduction electron to a $\pi$ band as expected in a rigid band model. But this model is clearly insufficient to explain all the measured profile difference features which reflect the total electronic change in intercalation.
\end{abstract}

\section{Introduction.}

When diffusing lithium atoms in graphite, the layer structure of the graphite host is conserved : in the first stage lithium intercalated graphite $\left(\mathrm{LiC}_{6}\right)$, each lithium layer is between two carbon layers. The stacking sequence of carbon atoms, $\mathrm{A}-\mathrm{B}$ in graphite, is modified to $\mathrm{A}-\mathrm{A}$ in $\mathrm{LiC}_{6}$. The physical properties of $\mathrm{LiC}_{6}$ are very different from those of graphite [1]; for instance the anisotropy of the in-plane to $c$-axis conductivity is three orders of magnitude smaller, which means that $\mathrm{LiC}_{6}$ can hardly be considered as a bi-dimensional material, which is generally admitted for graphite. Many experiments have been made to determine the electronic structure modification introduced by the presence of the $\mathrm{Li} 2 \mathrm{~s}$ electron [2], and X-ray inelastic scattering is a very suitable tool for studying such delocalized electrons. So we compared the $\mathrm{LiC}_{6}$ Compton profile to the graphite one under the same experimental conditions. 
Recently a self-consistent pseudopotential electronic structure calculation has been carried out for $\mathrm{LiC}_{6}$ by Holzwarth et al. [3] and a charge density has been derived. To estimate the role played in the intercalation compound by the outer $\mathrm{Li}$ electron, they make the difference between $\mathrm{LiC}_{6}$ and $\mathrm{C}_{6}$ densities $\left(\mathrm{C}_{6}\right.$ is actually a graphite with $\mathrm{A}-\mathrm{A}$ stacking sequence) and establish that the total electron difference density (between $\mathrm{LiC}_{6}$ and $\mathrm{C}_{6}$ ) is a $\pi$ density distorted in the direction of the $\mathrm{Li}$ ions. Within the same method a charge density calculation has been performed on pristine graphite as well [4].

Previous measurements have been performed on pyrolitic graphite using $\gamma$-ray inelastic scattering by Reed et al. [5] and by Paakari [6], both with a low resolution. They exhibit large discrepancies with pseudopotential and molecular orbital calculations by Reed et al. [5]. In principle, similar information can be obtained by angular correlation profiles from positron annihilation but as Berko et al. have shown [7], a quantitative information is difficult to extract due to the preferential positron annihilation with the $\pi$ electrons.

We present here the first Compton profile measurement on $\mathrm{LiC}_{6}$ and, in the discussion, we will compare the result with the measurement of Cartier et al. [8] by positron annihilation.

\section{Experimental method.}

The Compton profile $J\left(p_{z}\right)$ is proportional to the one-dimensional electron momentum density of the scatterer, i.e.

$$
J\left(p_{z}\right)=\sum_{i} \int_{p_{x}} \int_{p_{y}} n_{i}(\mathbf{p}) \mathrm{d} p_{x} \mathrm{~d} p_{y}
$$

where the sum is over all the electrons, $n_{i}(\mathbf{p})$ is the momentum density for the electron $i$

$$
n_{i}(\mathbf{p})=\chi_{i}(\mathbf{p}) \chi_{i}^{*}(\mathbf{p})
$$

with $\chi_{i}(\mathbf{p})$ the momentum space wave function and

$$
q=p_{z}=\mathbf{k} \cdot \mathbf{p} / k
$$

the projection of the momentum p on the scattering vector $\mathbf{k}$. Therefore the spectral analysis is an accurate test for wave functions, especially for valence and conduction electrons, since they are well localized in the momentum space. Moreover, this method, insensitive to defects, is particularly suitable for less organized materials. As it appears from equation (1), the electron density is integrated on planes perpendicular to the scattering vector; the experimental set-up is choosen so that $\mathbf{k}$ is parallel or perpendicular to the $c$-axis.

Compton profiles have been measured with the use of a three axis focusing spectrometer described elsewhere $[9,10]$. The synchrotron radiation beam of LURE-DCI is monochromatized to provide $12.858 \mathrm{keV}$ photons in a $15 \mathrm{eV}$ bandwidth. The photons scattered at an angle of $137^{\circ}$ are energy analysed through a curved crystal and are collected with a position sensitive detector. The full width at half maximum of the measured resolution is $25 \mathrm{eV}$ i.e. 0.15 atomic unit (a.u.) of momentum; this has to be compared with the 0.46 a.u. resolution in $\gamma$-ray experiments.

To keep the same data processing, the same geometry for the apparatus and the samples is conserved. No correction has been made for the resolution function. Because we compare profiles, all the isotropic processes i.e. multiple scattering [11], electron-electron correlations [12], impulse approximation deviation, are not to be taken into account. 


\section{Samples.}

Two Highly Oriented Pyrolitic Graphite (HOPG) samples, with orientational deviations along the $c$-axis around $0.5^{\circ}$, are used to study the electron density parallel to the $c$-axis and the average in the basal plane. They are $0.8 \times 1.5 \times 0.5 \mathrm{~cm}$ with the largest surface respectively perpendicular and parallel to the $c$-axis. A third sample has been intercalated by heating graphite and lithium at $400^{\circ}$ in a copper tube according to the method described elsewhere [13] ; it allows us to measure the Compton profile with the scattering vector along the ${ }_{i}$-axis. It was kept in a sealed cell with a beryllium window, under dry argon.

To be sure we did not get a second stage compound, we measured the 001 reflections with a $\theta-2 \theta$ spectrometer, before and after the experiment. The bright yellow colour was an extra proof of the stage, and the evidence of no presence of lithium at the surface.

\section{Experimental results.}

$\mathrm{A} \mathrm{LiC}_{6}$ directional Compton profile is shown in figure 1. For a photon energy loss equal to the $\mathrm{C}$ atom 1 s binding energy ( $284 \mathrm{eV}$, i.e. $-1.6 \mathrm{a}$ a.u.) we observe a clear slope discontinuity corresponding to the core profile departure. The Li edge departure $(55 \mathrm{eV})$ is too small to be evidenced. The Hartree-Fock calculated [14] 1s core electrons profile reported on the same figure starts just after the thresholds.

The graphite anisotropy for positive values of $q$ is reported in figure 2. It is compared with $\gamma$-ray results [5, 6] and with the two calculated ones [5]. For the scattering vector parallel to the $c$-axis, we compare the Compton profiles obtained on $\mathrm{LiC}_{6}$ and graphite in figure 3 . The graphite profile is normalized to the four valence and conduction electrons of $\mathrm{C}$ atom while the $\mathrm{LiC}_{6}$ profile is normalized to $1 / 6$ electron more. In such a way, the difference between the two profiles is the profile of the $\mathrm{Li}$ excess electron fraction by $\mathrm{C}$ atom.

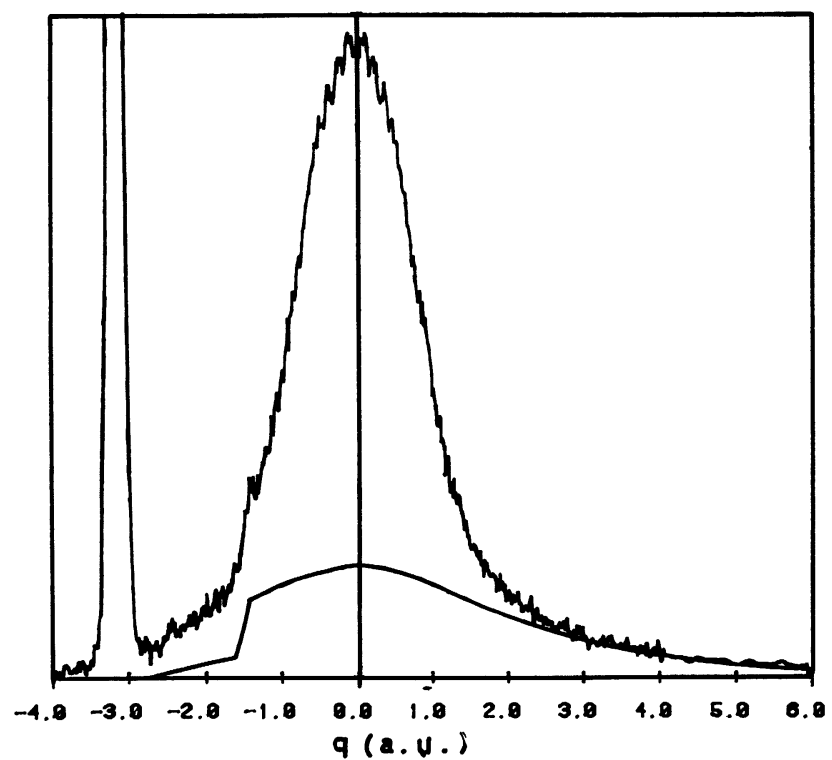

Fig. 1. - All electron $\mathrm{LiC}_{6}$ Compton profile. The raw curve is the experimental profile. For large $q$ it fits with the core-electron calculated one. The thermal peak is around -3.0 a.u. 


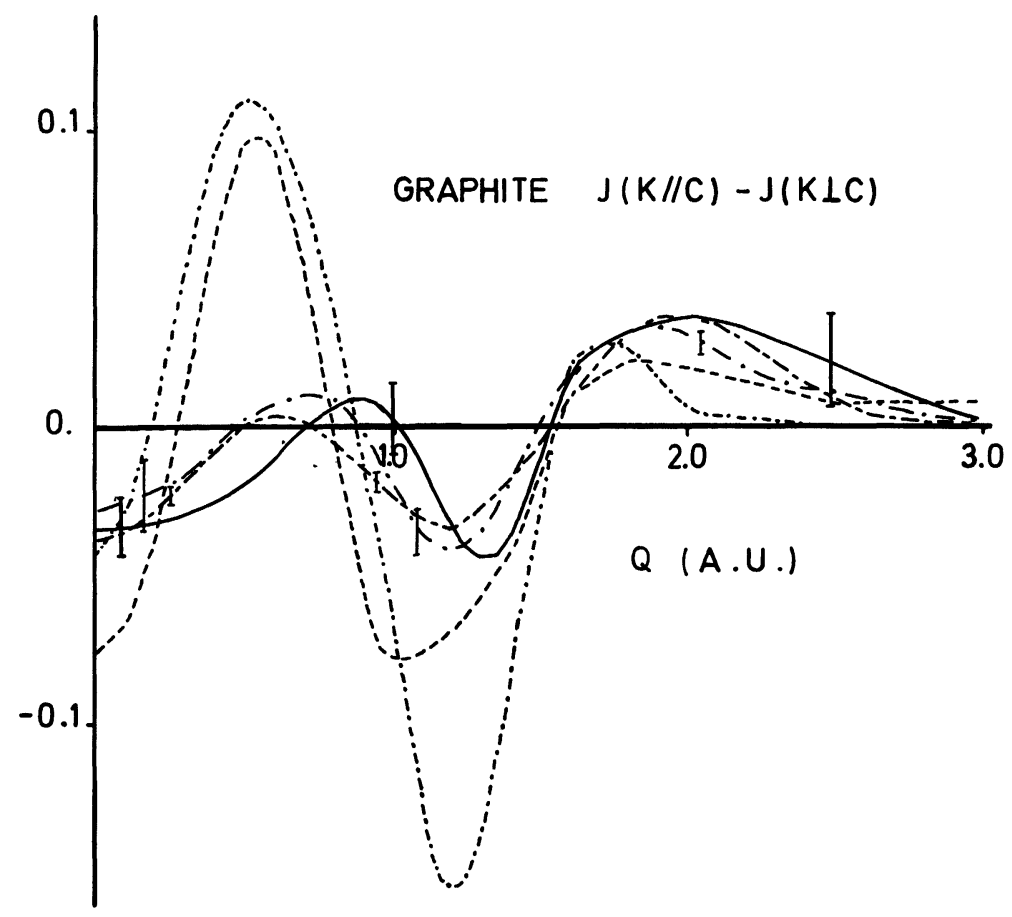

Fig. 2. - Graphite anisotropy. $J(q)$ for $\mathbf{k}$ parallel to the $c$-axis minus $J(q)$ for $\mathbf{k}$ perpendicular to $c$. Present experiment (-), $\gamma$-ray experiment by Reed et al. [5] (-•-), $\gamma$-ray experiment by Paakari [6] (- - -), pseudopotential calculation (-.-.--) and molecular orbital calculation (- - - -) by Reed et al. [5].

\section{Discussion.}

For the graphite anisotropy (Fig. 2), we observe a good general agreement between our experiment and $\gamma$-ray ones by Paakari [6] and by Reed et al. [5].

Near the Fermi momentum, all curves present a minimum but the comparison is difficult because the authors used a different graphite (pyrolitic). There is a large discrepancy with the available calculations in particular near $q=0.5$ a.u. and 1 a.u. As pointed out by Reed $e t$ al., they are respectively the lattice parameter and the interlayer distance in the momentum space and these authors conclude to the " inaccurate treatment of interplane bonding ". In the molecular orbital calculation, this bonding is not even taken into account. So the discrepancy with both models points to the huge effect of the interlayer interaction (i.e. of the stacking geometry). The Compton profile obtained from the self consistent pseudopotential [15] is the best theoretical fit obtained so far.

The electron momentum difference between $\mathrm{LiC}_{6}$ and graphite exhibits a maximum out of zero momentum ; as calculated by Berko [7] the graphite $\pi$ Compton profile for $\mathbf{k}$ parallel to the $c$ axis shows a maximum out of zero momentum and is null at zero (Fig. 3). In contrast, a metallike electron profile has a maximum at zero and collapses rapidly. So our experimental results cannot be totally explained by any of these opposite models. In particular, in the rigid band model, which is a good first approximation, the bands of graphite are folded in the Brillouin zone of $\mathrm{LiC}_{6}$ with an increase of the Fermi energy so that the excess electron is totally transferred to a graphite $\pi$ band : hence the density difference in this model is a graphite $\pi$ profile. The measured difference actually results from the total variation of outer (both valence and conduction) electron 

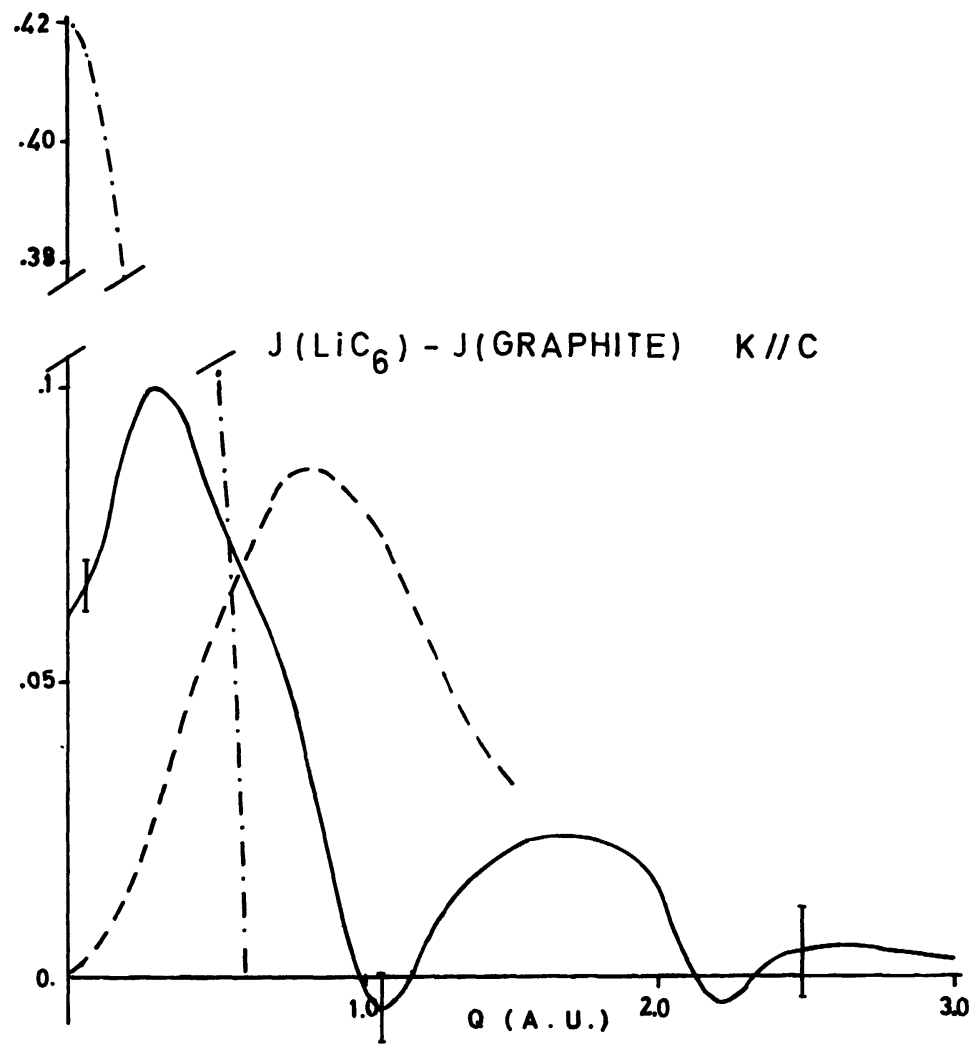

Fig. 3. - Compton profile difference for $\mathbf{k}$ parallel to $c, J\left(\mathrm{LiC}_{6}\right)$ minus $J$ (graphite). Our experiment $(-)$ is compared to a graphite Compton profile [7] (- - ) and to a metal-like profile (-. - ). All curves are normalized to $1 / 6$ electron.

density. Two facts are to be taken into account :

(i) introduction of an excess outer electron;

(ii) modification of the graphite charge distribution, due to the presence of the partially or totally ionized lithium, that can be illustrated in particular by the stacking change. Hence, the carbon valence electrons have to be described by different wave functions in $\mathrm{LiC}_{6}$ and graphite, taking into account both the charge distortion and the change in the adjacent layers interaction.

The theoretical maps of valence and conduction density difference between $\mathrm{LiC}_{6}$ and $\mathrm{C}_{6}$ show that the band distortion due to the $\mathrm{Li}$ ion only appears for the valence part [3]. In our difference profile (Fig. 3), the non zero momentum maximum is typical of a $\pi$ profile. The contribution at zero momentum can be attributed to a $2 \mathrm{~s} \mathrm{Li} \mathrm{participation} \mathrm{that} \mathrm{is} \mathrm{hybridized} \mathrm{with} \mathrm{the} \mathrm{initial} \mathrm{gra-}$ phite orbital in the $\mathrm{LiC}_{6}$ valence orbitals. The peak centre is shifted towards low momentum in comparison with the $\pi$ graphite, which means that the valence and conduction electrons are more delocalized than with the rigid band model. This is consistent with the larger extension of the $\mathrm{LiC}_{6}$ $\pi$ density compared to the undistorted graphite $\pi$ one (Fig. 5 of Ref. [3]) and with the particular sensitivity of the profile near $q=1.0$ a.u. to interlayer bonding. The Compton profile [15] derived from this model [3] presents the same general features as our measured one.

The positron annihilation of Cartier et al. [8] does not exhibit for $\mathrm{LiC}_{6}$ a narrow component like in a heavy alkali metal $\left(\mathrm{KC}_{8}\right)$ " which strongly supports the existence of an alkali-like electron 
gas $"$. Nevertheless they notice that the angular correlation profile, for $q$ parallel to $c$-axis, is higher than the HOPG one at zero. Both the absence of a narrow component and the enhancement of the profile at zero momentum show a qualitatively good agreement between the results of the two methods.

In conclusion, the zero contribution in the measured difference profile shows that the rigid band model is not accurate enough, thus it is necessary to consider both the charge transfer and the modification of the valence bands.

\section{Acknowledgments.}

We wish to thank Drs M. Y. Chou, S. G. Louie, M. L. Cohen and N. A. W. Holzwarth for permission to cite their work prior to publication and for fruitful discussions. We are grateful to Dr. Y. Petroff for his constant interest to the subject. We are indebted to Dr. A. W. Moore from Union Carbide who gave us the HOPG sample. We also wish to acknowledge the Laboratoire de l'Accélérateur Linéaire in Orsay and Drs P. Marin and M. P. Level for providing the synchrotron beam.

\section{References}

[1] Dresselhaus, M. S. and Dresselhaus, G., Adv. Phys. 30 (1981) 139.

[2] Solin, S. A., Physica 105B (1981) 481.

[3] Holzwarth, N. A. W., Louie, S. G. and Rabil, S., Phys. Rev. B 28 (1983) 1013.

[4] Holzwarth, N. A. W., Louie, S. G. and Rabil, S., Phys. Rev. B 26 (1982) 5382.

[5] Reed, A. W., Eisengerger, P., Pandey, K. C. and Snyder, L. C., Phys. Rev. B 10 (1974) 1507.

[6] PaAkari, T. L. P., Phys. Fenn. 9 (1974) 185.

[7] Berko, S., Kelley, R. E. and Plaskett, J. S., Phys. Rev. 106 (1957) 824.

[8] Cartier, E., Heinrich, F., Pfluger, P. and Guntherodt, H. J., Solid State Commun. 38 (1980) 985.

[9] Loupias, G., Petiau, J., J. Physique 41 (1980) 265.

[10] Loupias, G., Petiau, J., Issolah, A. and Schneider, M., Phys. Status Solidi B 102 (1980) 79.

[11] ChOMILIER, J., Distribution des impulsions électroniques du graphite et d'un composé d'insertion ( $\left.\mathrm{LiC}_{6}\right)$, Thèse de troisième cycle Université Paris 6 (1982).

[12] Chou, M. Y., Lam, P., Cohen, M. L., Loupias, G., Chomilier, J. and Petiau, J., Phys. Rev. Lett. 49 (1982) 1452.

[13] Guérard, D., Herold, A., Carbon 13 (1975) 337.

[14] Weiss, R. J., Harvey, A. and Phillips, W. C., Philos. Mag. 17 (1968) 241.

[15] Chou, M. Y.. Louie, S. G., Cohen, M. L. (Department of Physics, University of California, Berkeley, California) and Holzwarth, N. A. W. (Wake Forest University, Winston Salem, North Carolina) private communication. 